\section{Improved Corrosion Resistance of Plasma Carbon Coated NiTi Orthopedic Materials}

\author{
R. W. Y. Poon ${ }^{1}$, X. Y. Liu ${ }^{1}$, C. Y. Chung ${ }^{1}$, P. K. Chu ${ }^{1 *}$, \\ K. W. K. Yeung ${ }^{2}$, W. W. Lu' ${ }^{2}$, and K. M. C. Cheung ${ }^{2}$ \\ ${ }^{1}$ Department of Physics \& Materials Science, \\ City University of Hong Kong, Kowloon, Hong Kong; \\ ${ }^{2}$ Department of Orthopedic Surgery, University of Hong \\ Kong, Pokfulam, Hong Kong
}

Nickel titanium (NiTi) alloys are useful in orthopedic applications because of their super-elastic properties and shape memory effects. However, when NiTi is used for a prolonged period of time, harmful $\mathrm{Ni}$ ions can leach out into the surrounding body fluid inside a human body, and so it is important to design a method to impede the out-diffusion of nickel from the materials into the biological medium. We aim at producing a barrier to mitigate the release of $\mathrm{Ni}$ ions during normal use. Carbon coatings have been shown to possess excellent biocompatibility and good mechanical strength. In this work, amorphous hydrogenated DLC films with a graded C/NiTi interface were fabricated by plasma immersion ion implantation \& deposition (PII \& D) to provide such a barrier layer on NiTi. The elemental depth profiles and film thickness were determined by $\mathrm{X}$-ray photoelectron spectroscopy (XPS) whereas the surface morphology was evaluated using atomic force microscopy (AFM). The film structure was studied by $\mathrm{X}$-ray diffraction (XRD) and Raman spectroscopy. The corrosion resistance of the film was investigated using electrochemical tests based on ASTM G5-94. Compared to the control sample, the corrosion potential of the sample with the carbon coating changes from -250 to $-50 \mathrm{mV}$ and the film breakdown potential increases from 250 to $1200 \mathrm{mV}$. The corrosion current also diminishes from $10^{-6}$ to $10^{-7}$ A. The simulated body fluid (SBF) solutions after the electrochemical test were analyzed for $\mathrm{Ni}$ concentrations by inductively-coupled plasma mass spectrometry (ICPMS) and that data show that a much smaller amount of $\mathrm{Ni}$ has been released from the treated sample surface compared to the untreated control sample surface. Our results thus indicate that the deposited DLC film is effective in retarding the release of $\mathrm{Ni}$ ions from the bulk materials and more superior corrosion resistance is achieved based on our tests in a simulated fluid medium and at human body temperature.

\section{Rat Osteoblasts Growth on Surface of Plasma-Sprayed Bioceramic Coatings}

\author{
X. Y. Liu ${ }^{1,2}$, C. X. Ding ${ }^{1}$, and P. K. Chu ${ }^{2 *}$

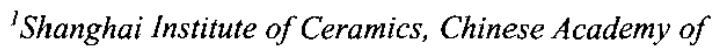 \\ Sciences, 1295 Dingxi Road, Shanghai 200050, China; \\ ${ }^{2}$ Dept. of Physics \& Materials Science, City University of \\ Hong Kong, Kowloon, Hong Kong
}

$\mathrm{Al}_{2} \mathrm{O}_{3}, \mathrm{ZrO}_{2}, \mathrm{TiO}_{2}$ and wollastonite coatings were deposited onto Ti-6Al-4V substrates employing atmospheric plasma spraying. Rat osteoblasts were subsequently seeded onto the surface of the coatings to evaluate the growth behavior of osteoblasts on the different materials. Scanning electron microscopy (SEM) was used to evaluate the morphologies of the osteoblasts seeded onto the surface of the coatings for different duration of time. Our data indicate that it is difficult for the osteoblasts to survive and proliferate on the $\mathrm{Al}_{2} \mathrm{O}_{3}$ surface showing that this coating has very poor biocompatibility. Osteoblasts can, however, survive and proliferate slowly on the $\mathrm{TiO}_{2}$ and $\mathrm{ZrO}_{2}$ coating surface indicating that these two coatings have certain cytocompatibility while they cannot accelerate the proliferation very well on the surface of the wollastonite coating. Therefore, the biocompatibility of wollastonite coating is better than that of $\mathrm{Al}_{2} \mathrm{O}_{3}, \mathrm{TiO}_{2}$ and $\mathrm{ZrO}_{2}$ coatings. 